\title{
Clinical Psychological Science
}

\section{Measuring the Severity of Negative and Traumatic Events}

David C. Rubin and Nicole Feeling

Clinical Psychological Science published online 10 April 2013

DOI: $10.1177 / 2167702613483112$

The online version of this article can be found at:

http://cpx.sagepub.com/content/early/2013/04/09/2167702613483112

Published by:

(४) SAGE

http://www.sagepublications.com

On behalf of:

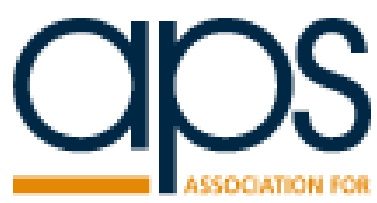

PSYCHOLOGICAL SCIENCE

Association for Psychological Science

Additional services and information for Clinical Psychological Science can be found at:

Email Alerts: http://cpx.sagepub.com/cgi/alerts

Subscriptions: http://cpx.sagepub.com/subscriptions

Reprints: http://www.sagepub.com/journalsReprints.nav

Permissions: http://www.sagepub.com/journalsPermissions.nav

>> OnlineFirst Version of Record - Apr 10, 2013

What is This? 


\title{
Measuring the Severity of Negative and Traumatic Events
}

Clinical Psychological Science

XX(X) 1-15

(C) The Author(s) 2013

Reprints and permissions:

sagepub.com/journalsPermissions.nav

DOI: $10.1177 / 2167702613483112$

cpx.sagepub.com

\author{
David C. Rubin and Nicole Feeling \\ Duke University
}

\begin{abstract}
We devised three measures of the general severity of events, which raters applied to participants' narrative descriptions: (a) placing events on a standard normed scale of stressful events, (b) placing events into five bins based on their severity relative to all other events in the sample, and (c) an average of ratings of the events' effects on six distinct areas of the participants' lives. Protocols of negative events were obtained from two nondiagnosed undergraduate samples ( $n=688$ and 328), a clinically diagnosed undergraduate sample, all of whom had traumas and half of whom met posttraumatic stress disorder (PTSD) criteria $(n=30)$, and a clinically diagnosed community sample who met PTSD criteria $(n=75)$. The three measures of severity correlated highly in all four samples but failed to correlate with PTSD symptom severity in any sample. Theoretical implications for the role of trauma severity in PTSD are discussed.
\end{abstract}

\section{Keywords}

posttraumatic stress disorder, severity, A criterion

Received 11/17/12; Revision accepted 2/22/13

Events severe enough to meet the A criterion of posttraumatic stress disorder (PTSD) can lead to PTSD. No other diagnosis, except for acute stress disorder, hinges on the classification of events. To qualify for the diagnosis in the third edition of the Diagnostic and Statistical Manual of Mental Disorders (DSM-III; American Psychiatric Association, 1980, p. 236), events had to be severe enough to meet the A criterion by being "generally outside the range of usual human experience" and evoking "significant symptoms of distress in most people." Moreover, there was an implied continuum of kinds of events: "Some stressors frequently produce the disorder (e.g., torture) and others produce it only occasionally (e.g., car accidents)." Later, in the DSM-IV-TR, the type of severity as defined by the A criterion was restricted to those events in which "the person experienced, witnessed, or was confronted with an event or events that involved actual or threatened death or serious injury, or a threat to the physical integrity of self or others" (American Psychiatric Association, 2000, p. 467). Most frequently, events severe enough to meet the A criterion do not lead to PTSD (Breslau, Davis, Andreski, \& Peterson, 1991; Kessler, Sonnega, Bromet, Hughes, \& Nelson, 1995). Nonetheless, researchers have consistently noted that the symptoms of PTSD can be produced by events whose severity does not meet the A criterion and that such less severe events can produce an even higher level of symptom severity than those that do meet the criterion, often in the same sample of individuals (Dohrenwend, 2010; Lancaster, Melka, \& Rodriguez, 2009; McNally \& Robinaugh, 2011; Rubin, Berntsen, \& Bohni, 2008).

The purpose of this article is to explore objective measures of general severity and their ability to predict PTSD symptom severity. To do this, we examine a broad range of negative events; evaluating severity in relation to the A criterion logically requires both A-criterion and non-Acriterion events (see Rubin, Berntsen, et al., 2008, p. 988). The most useful diagnostic outcome of this study of event severity would be if we could define a continuum of event severity and that events that exceed a particular level of severity were needed for, or were more likely to produce, a diagnosis of PTSD. We know of no attempt at this empirical approach. Rather, two distinct empirical

\section{Corresponding Author:}

David C. Rubin, Duke University, Department of Psychology \& Neuroscience, Box 90086, Durham, NC 27708-0086

E-mail: david.rubin@duke.edu 
approaches exist in the literature (Breslau, 2012). The first is to examine different categories of events such as childhood abuse or sexual assault and see if they are more likely to cause PTSD (e.g., Brewin, Andrews, \& Valentine, 2000; Dedert et al., 2009; Kessler et al., 1995). The second is to limit the investigation to a particular category of events where objective severity can be inferred, such as distance from the wave in a tsunami or from the epicenter of an earthquake, and measure these effects (e.g., Berntsen \& Rubin, 2008; Brewin et al., 2000). Both of these empirical approaches have had some success in predicting PTSD. However, if they are to lead to a measure that can be applied to all events, the first would require that we place all events in categories that have an overall severity rating, and the second would require that we devise ways of applying it to more situations. Moreover, if both approaches are valid, to arrive at a prediction of PTSD symptom severity, they would need to be combined quantitatively (Weiss et al., 2010). Thus, it seems simpler to attempt to formulate a general measure of event severity.

Our approach is to start by defining what appears on the surface to be conceptually and methodologically distinct measures of the general severity of events and examine whether the measures are related to each other. To the extent that these distinct measures converge on the same empirical measure, it will be more likely that we are tapping an underlying concept of the general severity of events. After evaluating this, we investigate whether these measures predict PTSD symptom severity.

The need for a single event severe enough to be considered a trauma in the diagnosis of PTSD and the role of one or more severe negative events in our current theoretical understanding of PTSD assume an understanding of event severity that is at least up to the task of defining such events. The lack of a theory or clear empirical support for measures of how severe different events need to be to cause the symptoms of PTSD threatens the logic of the diagnosis, though not the reality of the suffering that accompanies PTSD. To advance our understanding, we need either to find a more predictive measure of event severity or to develop other theoretical explanations for the symptoms that arise in PTSD. This article is one attempt.

\section{Measuring the Severity of Events as Rated by Neutral Observers}

It is extremely difficult to measure objective severity in an analytic or mechanical way. For example, the objective distance from the epicenter of an earthquake does not note how dangerous an individual situation was, including the exact location or type of building the person was in. Moreover, the severity of the potential loss varies with the individual in ways that do not depend on personality or other general factors we can easily measure. A "minor" injury to the hand of a musician or surgeon that restricts motion by a quantifiable amount may be more severe than the same injury to another person. Thus, there is no ideal, purely analytic objective measure. In more literary terms, we lack the view of the all-knowing neutral narrator recording the event. In addition, for practical and ethical reasons, there is rarely a report made at the time of the trauma from either the person experiencing the event or a neutral observer. What we usually have is the memory reported by the person a considerable time after the trauma; for diagnosis the report occurs at least a month after the trauma. One might assume that the legal system would offer metrics of severity for claims of damages, but for PTSD it defers to mental health expert witnesses and the evaluation of their expert testimony by judges or jurors (Smith, 2011).

Faced with this problem, we do what psychologists usually do and use raters' judgments to measure concepts we cannot define analytically: in this case, determining the general severity of a traumatic event given its narrative description. This has two problems. The first problem is that we need to assume some cultural similarity among the participants and raters, and so we try to draw the raters from a culture similar to that of the participants. The second problem is that the narrative descriptions could be influenced by the severity the participants assigned to the events. The result would be that our ratings of the severity of the event in the memory would already include some of the effects of the participants' perceptions and memory distortions. Thus, any biases caused by the participants stressing particular aspects of their events would be in the known direction of attributing the effects of individual differences and other factors that cause memory distortions to event severity. This could make our ratings of event severity more predictive than ratings of truly objective descriptions of the event. We cannot avoid this completely but can alert the raters to it and have them judge, as best they can, the actual situation and not the emotional reactions and extraneous descriptions.

We include four kinds of ratings of severity classified by their being rated by either a neutral observer or the participant crossed with their being either a measure of general severity or the specific measure of severity used by the $D S M-I V$. Our main theoretical interest is the neutral observer measures of general severity. We chose our three neutral observer measures of general severity to be as different on the surface conceptually and mechanically as we could. In this way any agreement among them would be an empirical finding and not caused by similarity in wording or methods; that is, if we find a single concept of event severity, it would be from distinct 
measures. First, we had a rating of Versus Other Events severity, in which we asked judges to decide the severity of events relative to all the events in the sample by placing them into five bins based on how an average person would categorize each event from least objectionable (1) to events they would most like to avoid (5). Second, the raters decided what number each event would have if it were placed among the normed stressful items of the Holmes-Rahe Scale (Holmes \& Rahe, 1967). Third, we calculated an average of 7-point ratings of Six Kinds of Damage that an event could have, including how physically, financially, and emotionally damaging the event would be to the person and how much it would affect the person's future.

Individually, all general severity measures have clear advantages and limitations. However, as a set they represent a variety of approaches to defining severity. Moreover, as a set they are less subject to the critique that they fail to measure severity as it is commonly understood if, as will be shown, they correlate highly with each other.

We also included an other-rated measure based on the A1 criterion of the DSM-IV for the narrative descriptions of Studies 1 and 2, which did not have clinically diagnosed samples. Respondents in Studies 3 and 4 had clinical diagnoses and thus had interview assessments of the A criterion. Unlike the three measures of general severity, the A1 is a measure of the particular kind of severity that is needed for a diagnosis of PTSD. We considered adding other-rated judgments of the A2 criterion, but they were too difficult with the information given in the narratives because they require a judgment of the person's emotions, which was rarely given. Our raters for the A1 were experienced in administering the Clinician-Administered PTSD Scale (CAPS; Blake et al., 1995; Weathers, Keane, \& Davidson, 2001).

To contrast to these other-rated measures of severity, we included self-rated measures formulated to be as similar to the other-rated measures as possible. That is, the participants were not asked to rate how they felt or reacted to the event, but rather to rate objective severity of the event as they would be judged by another person. For the general severity measures, we were able to include measures based on the Versus Other Events and Kinds of Damage other-rated measures; the Holmes-Rahe measure was too complex to obtain from the participants. We were able to add self-rated A1 and A2 measures based on the DSM-IV-TR.

\section{Hypotheses}

We have three hypotheses. Our first is that our three other-rated measures of general severity, each of which has clear face validity, will correlate substantially. This is a simple enabling hypothesis. If it is not supported, the examination of the role of event severity becomes more complex because we will not be able to measure event severity as a unitary concept in our studies. However, if the hypothesis is supported, we will have introduced to the literature a set of three empirically supported, conceptually related measures of general event severity, something that is now absent and that would benefit future research. Our second hypothesis, which is tested most clearly in Study 2, is that our measures of general event severity correlate with similar measures of selfrated event severity. This is a simple test to demonstrate that our other-rated measures relate to the concept they are intended to measure, which would further support the validity of these measures. Our third hypothesis is that our measures of general event severity will predict PTSD symptom severity, at least to a modest degree, so that they will predict PTSD symptom severity both individually and in conjunction with our measures of neuroticism, depression, and the centrality of an event to a person's identity. Given the lack of findings of strong correlations between event severity as it has been measured and PTSD symptom severity, our support for this hypothesis is limited.

\section{Outline of the Four Studies}

We used two basic recruitment methods. For our first two studies, we sampled all undergraduates enrolled in introductory psychology courses who took part in a department-wide subject pool. Thus, we obtained a wide range of symptom severity, no clinical diagnosis, and a convenience sample similar to the ones on which most nonclinical research in psychology is undertaken. For our last two studies, we sampled university (Study 3 ) and general community (Study 4) participants with A-criterion traumas who were selected to meet the inclusion and exclusion requirements needed for studies of PTSD. The recruitment and screening of these participants produced samples that are less representative of a well-defined population, but they have the advantage of a clinical diagnosis. The large range of negative events we have in the first two studies, including those that are clearly not A traumas, should increase the effects of severity. Restricting the range of stressful events to only A traumas, as we do in the last two studies, should decrease the range and therefore the effects of severity, but it should allow us to examine severity in the range needed for a PTSD diagnosis. Thus, each sample has its weaknesses; using all four together ensures these individual weaknesses cannot produce our results.

To provide a more complete description of our samples and to ensure that our measure of PTSD symptom severity correlates with other standard measures as it usually does in the literature, we included several 
common tests. For all four studies we include the Beck Depression Inventory-II (BDI-II; Beck, Steer, \& Brown, 1996), the Centrality of Event Scale (CES; Berntsen \& Rubin, 2006, 2007), and the PTSD Checklist (PCL; Blanchard, Jones-Alexander, Buckley, \& Foneris, 1996; Weathers, Litz, Huska, \& Keane, 1994). To provide a description of personality measures, in Studies 1 and 2 we include the Big Five Inventory (BFI; John, Donahue, \& Kentle, 1991), and in Study 4 the NEO Personality Inventory (NEO; Costa \& McCrae, 1992). To provide a measure of the numbers of traumas the community clinical sample of Study 4 had, we included the Traumatic Life Events Questionnaire (TLEQ; Kubany et al., 2000).

\section{Study 1: First Undergraduate Sample Method}

Participants. A total of 688 Duke University undergraduates (440 women, 248 men, mean age of 19.16) enrolled in introductory psychology courses completed the questionnaires.

Materials. The BDI-II (Beck et al., 1996) is probably the most widely used test of general depression symptoms. The 21 items assess various emotional manifestations of depression including sadness, hopelessness, irritability, and guilt, and physical symptoms such as fatigue, weight loss, and lack of interest in sex. A tremendous amount of research attests to its internal consistency and validity.

The BFI (John et al., 1991) is a 44-item measure of the broad personality domains of extraversion, neuroticism, agreeableness, conscientiousness, and openness. Each domain is assessed by 8 to 10 short phrases (e.g., for conscientiousness, "Perseveres until the task is finished"). The internal consistency of the scales is high, and convergent and discriminant validities of the scales are well established (John \& Srivastava, 1999).

The CES (Berntsen \& Rubin, 2006, 2007) measures the extent to which a traumatic memory functions as a central component of a person's identity and life story. The short form of the CES, which we used, consists of seven items, which include "I feel that this event has become part of my identity. This event has become a reference point for the way I understand myself and the world. This event has permanently changed my life. I often think about the effect this event have will have on my future. This event was a turning point in my life."

When completing the PCL (Blanchard et al., 1996; Weathers et al., 1994), participants nominate a specific stressful event and rate on 5-point scales from not at all to extremely how much they have been bothered by it on each of 17 symptoms of PTSD identified in the
$D S M-I V-T R$. We obtained two measures from the PCL. The main measure of the severity of symptoms is the continuous measure of the sum of self-ratings of the 17 $D S M-I V$-TR symptoms on the PCL, which is probably the most commonly used measure of symptom severity in research studies. We also calculated a dichotomous measure somewhat closer to diagnostic status. We did this to check that the simple sum of PCL symptom ratings did not deviate empirically in important ways from the diagnostic procedure a clinician would follow, though here we have only unchecked self-reports. This measure of status, which is based on the DSM-IV-TR, has a value of 1 if the participant has one or more $\mathrm{B}$ (reliving) symptoms, three or more $\mathrm{C}$ (avoidance) symptoms, and two or more D (arousal) symptoms all rated as 3 (moderately bothered) or higher on the PCL 5-point scale of not at all bothered to extremely bothered.

Procedure. Our measures were part of a general webbased screening of the Department of Psychology and Neuroscience that is routinely completed at the beginning of each academic semester. Our instruments were placed among those of other researchers from the department. We had participants rate their own memories based on the $D S M-I V$ description of the A1 and A 2 criteria: for A1, "Did you experience, witness, or were you confronted with an event that involved actual or threatened death or serious injury, or threat to the physical integrity of yourself or others?"; for A2, "Did your response involve intense fear, helplessness, or horror?"

The order of our questions and instruments was as follows. Participants did the BFI after providing demographic information. Later in the session they were asked to "please take a moment to think of what negative event or experience from your life is most troubling and stressful to you now. Once you have nominated your most distressing event, please answer the questions that follow in reference to this event." They then recorded a brief description of the event. Immediately following this they completed the PCL. They were then asked to "please think back upon the negative event you nominated earlier and answer the following questions in an honest and sincere way." They then completed the CES and responded to the DSM- $I V-T R$ wording of the A1 and A2 criteria. The BDI-II occurred later in the session.

\section{Ratings}

The Versus Other Events rating of severity was produced by asking judges to decide the severity of events by placing them into bins as follows.

Please examine all the events in the set of events you are working on and divide them into bins 1 to 
5 so that each bin has about $1 / 5$ th of the events. It is not necessary to have exactly $1 / 5$ th, but each bin should have a minimum of about $15 \%$ and a maximum of about $25 \%$ of the events. Consider an average person. How do you think they (as opposed to the person recording the event) would categorize each event? Give the $1 / 5$ th of the events that they would most like to avoid a 5 . Give the next $1 / 5$ th a 4 , and so forth until the $1 / 5$ th of the events that are least objectionable is given a 1 .

The Holmes-Rahe Scale rating of severity was based on the 43 items of a widely used scale that measures overall life stressors, both positive and negative, not just trauma (Holmes \& Rahe, 1967). The highest stress of 100 is given to death of a spouse, marriage has a stress of 50 , and the lowest stress of 11 is given to minor violations of the law. We gave the scale to our judges and asked them to give each description a number that would place it at its best position within the scale or above the scale maximum of 100 if it was more stressful than the death of a spouse. Thus, we had people use an existing stress scale to provide a value for each description. The judges were instructed, "You do not have to agree with the values individually, but they should serve as a way for you to anchor your response."

The Six Kinds of Damage rating of severity is a composite of six kinds of "damage" that an event can have: physical damage to the person, physical damage to family or friends, the effect on the person's future, the emotional toll on the person, the toll on the person's family relationships, and the effect on the person's financial well-being. Each of these was rated on a 7-point scale from minimal to maximum possible. We initially also considered other possible scales that we thought might be reasonable and eliminated them because they could not be judged reliably on a pilot set of descriptions or because they were infrequent in our data. We did not eliminate any kind of damage because it failed to add to the internal consistency of the summed scale because having one kind of damage often makes other kinds of damage less likely.

The A1 severity was rated as closely as possible to the CAPS procedure, given it was made on narrative descriptions without any access to the participants themselves. Protocols were rated as probable A1, not A1, or unclear given the information available.

For the three measures of general severity, undergraduate raters were provided only with the subjects' descriptions of their events; they were blind to the PCL score and all other measures taken. They were also not informed of our hypothesis that their severity ratings would predict PTSD symptom severity at least to a moderate degree. The undergraduate raters received minimal training because the task was easy to explain. In addition to receiving brief verbal instructions, they rated pilot descriptions to ensure they understood the task. The same five raters did the Versus Other Events and Six Kinds of Damage ratings, which were done at the same time. Five different raters did the Holmes-Rahe Scale. It is important to note that the undergraduate raters were not trained in DSM-IV-TR diagnosis of PTSD and so were not biased by the A criterion in their ratings of severity.

The A1 dichotomy was done independently of the other rating by two raters who were experienced in administering the CAPS in a research setting at the Durham Veterans Affairs Medical Center. The two raters individually rated the protocols blind to other measures, and differences were resolved in discussions that included a third trained rater where interpretation of DSM-IV criteria was involved.

\section{Results}

The wide range of negative events described by the participants could be grouped into the following major categories: relationship issues including those with their family, 223; injury, illness, or accident to self (58) or others (50), 108; death of friend or family member, 91; school or career issues, 76; psychiatric disorder or addiction of self (31) or others (16), 47; unwanted sexual encounters including rape, 26; miscellaneous including victim of crime, trouble with law, terrorist attacks, sports, and difficulties with transitions, 121. There was excellent internal consistency in, and agreement among, the three other-rated general severity measures. The alphas for the individual scales are shown in Table 1. The alphas for our other-rated severity measures provide an index of how well the ratings done by our five raters and another sample of five raters drawn from the same population correlate with each other. They provide a measure directly comparable to the correlations of the other-rated severity measures with other variables that we report, providing an approximate upper limit on those correlations. For the Other-Rated A1 done by the two trained raters, the kappa was .72.

The Six Kinds of Damage severity correlated with the Versus Other Events and Holmes-Rahe severities at .90 and .88, respectively. The Versus Other Events and Holmes-Rahe severity correlated at .85 . The dichotomous Other-Rated A1 correlated with the Six Kinds of Damage, Versus Other Events, and Holmes-Rahe severity .43, .55, and .50 on the 508 descriptions that were complete enough to support this more difficult judgment (all $p s<$ .0001). The Other-Rated A1 also agreed well with the Self-Rated A1, $\chi^{2}(1)=121.80$, for comparison with other scales for which correlations were given, $r=.49$, ps < .0001 . Thus, we have reliable and converging measures 
Table 1. Study 1 Means and Correlations With PCL

\begin{tabular}{|c|c|c|c|c|c|}
\hline \multirow[b]{2}{*}{ Variable } & \multirow[b]{2}{*}{$M$} & \multirow[b]{2}{*}{$S D$} & \multirow[b]{2}{*}{$\alpha$} & \multicolumn{2}{|c|}{$r$ With PCL } \\
\hline & & & & Sum & Status \\
\hline \multicolumn{6}{|c|}{ Other-rated general severity } \\
\hline Versus Other Events & 2.93 & 1.23 & .92 & -.02 & .02 \\
\hline Holmes-Rahe Scale & 34.14 & 13.42 & .94 & -.02 & .01 \\
\hline Six Kinds of Damage & 1.49 & 1.12 & .97 & -.07 & -.02 \\
\hline \multicolumn{6}{|c|}{ Other-rated $D S M-I V$ severity } \\
\hline A1 & 0.11 & 0.31 & - & .01 & .02 \\
\hline \multicolumn{6}{|c|}{ Self-rated $D S M-I V$ severity } \\
\hline A1 & 0.30 & 0.46 & - & .03 & .04 \\
\hline $\mathrm{A} 2$ & 0.41 & 0.49 & - & $.25^{* * * * * *}$ & $.14^{* * * * *}$ \\
\hline \multicolumn{6}{|c|}{ Individual differences } \\
\hline CES & 2.65 & 1.14 & .93 & $.53^{* * * * * * *}$ & $.33^{* * * * * * *}$ \\
\hline BDI-II & 5.10 & 5.95 & .89 & $.49^{* * * * * * *}$ & $.40^{* * * * * * *}$ \\
\hline Big Five N & 23.10 & 6.06 & .85 & $.43^{* * * * * * * *}$ & $.31^{\text {********* }}$ \\
\hline Big Five E & 26.34 & 6.54 & .90 & -.05 & -.07 \\
\hline Big Five $O$ & 34.59 & 5.98 & .80 & -.00 & -.03 \\
\hline Big Five A & 33.51 & 5.47 & .80 & $-.20 * * * * * *$ & $-.17^{* * * * * * *}$ \\
\hline Big Five C & 32.50 & 5.80 & .84 & $-.17^{* * * * * *}$ & $-.15^{\text {******* }}$ \\
\hline PCL sum & 30.66 & 11.55 & .92 & - & $.70^{* * * * * * * * *}$ \\
\hline PCL status (\%) & 13.01 & 33.66 & - & $.70^{* * * * * * * * *}$ & - \\
\hline
\end{tabular}

of severity from three different perspectives and a moderate agreement with the Other-Rated A1. Moreover, the sum and status PCL measures correlated with the individual differences measures in reasonable ways, as shown in Table 1.

As in an earlier study with a similar population (Rubin, Boals, \& Berntsen, 2008), the Self-Rated A1 did not correlate with the PCL, though the Self-Rated A2 did. In our undergraduate samples, as well as in many studies comparing A-criterion and non-A-criterion negative events, as noted in the introduction, the A-criterion events often do not lead to differences in symptom severity and on occasion lead to lower symptom severity. Thus, the lack of correlation of the Self-Rated A1 with the PCL here is consistent with earlier studies. In contrast, the Self-Rated A2 does correlate with the PCL. In the proposed DSM-5 (http://www.dsm5.org/ProposedRevisions/Pages/ proposedrevision.aspx?rid=165, October 6, 2012), the A2 is no longer part of the A criterion, but persistent fear and horror, along with other negative emotions, form a symptom of negative alterations in cognitions and mood. It may function that way here, with current emotions being included as retrospective self-reports of emotions present at the event and thus correlating with the other symptoms of the PCL, or it could be that fear, horror, and helplessness accompany many severe events.

In spite of all measures correlating in reasonable ways, the other-rated severity measures did not predict either the PCL sum or the PCL status measure.

\section{Discussion}

We tested a large sample of undergraduates not selected with regard to PTSD symptoms or the severity of their most troubling negative event and thus produced substantial variability on these dimensions representative of our undergraduates. PTSD symptom severity correlated as would be expected with individual differences measures, and the three different methods of estimating other-rated general severity correlated between .85 and .90 with each other. However, other-rated event severity did not predict PTSD symptom severity. Given the surprising lack of a correlation, we decided to replicate this finding on a new sample. To investigate whether our 
internally consistent measures of other-rated general severity were capturing something related to what participants themselves thought was severity, we added two parallel measures of self-rated severity.

\section{Study 2: An Undergraduate Sample With Self-Reports of Severity}

\section{Metbod}

Participants. A total of 328 Duke University undergraduates (210 women, 118 men, mean age of 19.09) enrolled in introductory psychology courses completed the questionnaires.

Procedure. The materials and general procedures were identical to those in Study 1 except as follows. Two of the measures rated by neutral observers were modified to also produce self-ratings of severity and were placed immediately after the self-rated A1 and A2 items. Corresponding to the Versus Other Events rating, in which neutral observers place narrative descriptions into five bins, participants rated the events using the following instructions: "Overall, I believe that if the event happened to most people, they would consider the severity of the event as: 1 - negligible to 7 - as much as any event I could imagine." We reduced the six kinds of other-rated damage to four rating scales, combining scales that separated damage to self and family or friends. The resulting four scales, which were rated on a 7-point scale from 1 (negligible) to 7 (as much as any event I could imagine), were as follows:

How much physical damage did the event do to you or others very close to you? How much emotional damage did the event do to you or others very close to you? How much financial damage did the event do to you or others very close to you? Overall, how much does this event affect your future?

To make the self- and other-rated severity measures parallel, we averaged our other-rating subscales of injury to self and injury to close relations and our other-rating subscales of damage to social relations to the individual and social relations to their family so that the resulting four other-rating subscale scales matched the four subscales the participants rated.

\section{Ratings}

The rating procedure was similar to that used in Study 1, though new raters were added. Six raters scored the Holmes-Rahe and Four Kinds of Damage at the same time; three of these raters also scored the Versus Other Events scale a minimum of 2 weeks after the other two scales and blind to their earlier ratings. Three different raters also scored the Versus Other Events scales. The Other-Rated A1 was again scored by the same two clinically experienced raters who did these ratings in Study 1. Thus over the first two studies, the Holmes-Rahe, Versus Other Events, and Other-Rated A1 scales were each rated separately from the other scales, ensuring that correlations among our scales could not be due solely to a carryover of a general impression of severity.

\section{Results}

Using the same categories as Study 1, the negative events described by the participants could be grouped into the following major categories: relationship issues including those with their family, 96; injury, illness, or accident to self (48) or others (29), 77; death of friend or family member, 54; school or career issues, 27; psychiatric disorder or addiction of self (13) or others (7), 20; unwanted sexual encounters including rape, 9; miscellaneous, 45. There was excellent internal consistency in, and agreement among, the other-rated severity measures. The alphas for the individual scales are shown in Table 2. For the Other-Rated A1, kappa was .81. The Four Kinds of Damage severity correlated with the Versus Other Events and Holmes-Rahe severity at .89 and .92 , respectively. The Versus Other Events and Holmes-Rahe severity correlated at .88. Thus, we have converging measures of general severity from three different perspectives. The dichotomous Other-Rated A1 correlated with the Four Kinds of Damage, Versus Other Events, and Holmes-Rahe severity at $.46, .58$, and .51 (all $p s<.0001$ ) on the 236 descriptions that were complete enough to support this more difficult judgment. As in Study 1, the PCL correlated with the individual differences measures in reasonable ways. As in Study 1, the Other-Rated A1 also did not correlate with the PCL, nor did the Self-Rated A1, though again the Self-Rated A2 did. Thus, all measures correlated in reasonable ways, but as shown in Table 2, the otherrated severity measures did not predict the PCL sum. Unlike in Study 1, the PCL status measure correlated at .14 , .11, and .17 with the Holmes-Rahe, Four Kinds of Damage, and Other-Rated A1 severity, respectively (all $p s<.05)$. Given the relatively small magnitude of these correlations and their lack of significance in the same population and procedure in Study 1, little can be made of this difference beyond noting that there may be a small effect that is at the edge of our ability to detect with our current statistical power.

In contrast to the other-rated measures of severity, the self-rated measures of severity correlated with the PCL. It is hard to interpret these correlations in detail because 
Table 2. Study 2 Means and Correlations With PCL

\begin{tabular}{|c|c|c|c|c|c|c|c|}
\hline \multirow[b]{3}{*}{ Variable } & \multirow[b]{3}{*}{$M$} & \multirow[b]{3}{*}{$S D$} & \multirow[b]{3}{*}{$\alpha$} & \multicolumn{4}{|c|}{ Correlation with } \\
\hline & & & & \multicolumn{2}{|c|}{ PCL } & \multicolumn{2}{|c|}{ Self-rated } \\
\hline & & & & Sum & Status & $\begin{array}{l}\text { Four Kinds } \\
\text { of Damage }\end{array}$ & $\begin{array}{c}\text { Versus Other } \\
\text { Events }\end{array}$ \\
\hline \multicolumn{8}{|c|}{ Other-rated general severity } \\
\hline Versus Other Events & 3.02 & 1.24 & .95 & .06 & .10 & $.38^{* * * * * *}$ & $.49^{* * * * * *}$ \\
\hline Holmes-Rahe Scale & 34.13 & 14.69 & .95 & .10 & $.14^{*}$ & $.37^{* * * * * * *}$ & $.45^{* * * * * * *}$ \\
\hline Four Kinds of Damage & 1.54 & 1.08 & .98 & .05 & $.11^{*}$ & $.39 * * * * * * *$ & $.45^{* * * * * * * * 1}$ \\
\hline \multicolumn{8}{|c|}{ Self-rated general severity } \\
\hline Versus Other Events & 3.97 & 1.67 & - & $.29^{* * * * * * *}$ & $.25^{\text {******** }}$ & $.68^{* * * * *}$ & - \\
\hline Four Kinds of Damage & 2.92 & 1.25 & .69 & $.39^{* * * * * * * *}$ & $.32^{* * * * * * * *}$ & - & $.68^{* * * * * * * 1}$ \\
\hline \multicolumn{8}{|c|}{ Other-rated $D S M-I V$ severity } \\
\hline A1 & 0.15 & 0.36 & - & .11 & $.17^{*}$ & $.23^{* * * * *}$ & $.41^{* * * * * * *}$ \\
\hline \multicolumn{8}{|c|}{ Self-rated $D S M-I V$ severity } \\
\hline A1 & 0.35 & 0.48 & - & .03 & .06 & $.32 * * * * * *$ & $.40^{* * * * * * *}$ \\
\hline A2 & 0.45 & 0.50 & - & $.27^{* * * * * * *}$ & $.12^{*}$ & $.35^{* * * * * *}$ & $.35^{* * * * * * *}$ \\
\hline \multicolumn{8}{|c|}{ Individual differences } \\
\hline CES & 2.64 & 1.10 & .92 & $.54^{* * * * * *}$ & $.43^{* * * * * * *}$ & $.59 * * * * *$ & $.50 * * * * * *$ \\
\hline BDI-II & 5.81 & 7.12 & .92 & $.50^{0 * * * * * *}$ & $.43^{* * * * * *}$ & $.31^{1 * * * * * *}$ & $.14^{*}$ \\
\hline Big Five N & 2.90 & 0.78 & .84 & $.38^{* * * * * * *}$ & $.23^{* * * * * * *}$ & $.18^{* * * * * *}$ & .00 \\
\hline Big Five E & 3.34 & 0.76 & .87 & $-.13^{*}$ & -.11 & -.06 & -.03 \\
\hline Big Five $O$ & 3.51 & 0.63 & .82 & .01 & .02 & .06 & .06 \\
\hline Big Five A & 3.72 & 0.60 & .79 & 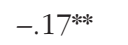 & -.08 & .02 & .08 \\
\hline Big Five C & 3.60 & 0.64 & .81 & $-.14^{*}$ & $-.15^{* * *}$ & -.01 & .09 \\
\hline PCL sum & 29.70 & 10.85 & .91 & - & $.71^{* * * * * *}$ & $.39^{* * * * * * *}$ & $.29^{* * * * * * *}$ \\
\hline PCL status (\%) & 13.11 & 33.80 & .91 & $.71^{* * * * * *}$ & - & $.32^{2 * * * * * * *}$ & $.25^{\text {******** }}$ \\
\hline Event age & 1136 & 1259 & - & -.08 & -.03 & .07 & .10 \\
\hline
\end{tabular}

Note: $n=328$, except for A1 other, for which $n=236$. PCL $=$ PTSD Checklist; CES $=$ Centrality of Event Scale; BDI-II $=$ Beck Depression Inventory-II; Big Five Inventory N, E, O, A, and C = neuroticism, extroversion, openness, agreeableness, and conscientiousness.

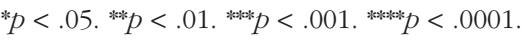

the measures were done at the same time, and the PCL ratings that were done first could have influenced the severity ratings, or they both could have been influenced by the participants' mood at the time. However, the correlations among the other-rated and self-rated severity are easier to interpret because they were done by different people. Although the Other-Rated Four Kinds of Damage, Other-Rated Versus Other Events, and OtherRated A1 did not correlate with the PCL, the self and other rated versions correlated at .39 for the Four Kinds of Damage scale, .45 for the Versus Other Events scale, and .61 for the A1. Alternatively for the A1, $\chi^{2}(1)=82.36$; all $p s<.0001$. Thus, the other-rated scales shared variance with the corresponding self-rated scales, just not the variance the self-rated scales shared with the PCL. This implies that our other-rated scales are capturing some aspects of the participants' severity ratings, even if the other-rated scales do not predict symptom severity.

For this sample, immediately after describing their stressful events, participants dated them using a $\mathrm{mm} / \mathrm{dd}$ / yyyy format. Participants were instructed to make their best estimate, reporting only the year if that was all they could do. From this we calculated how many days ago the event occurred to investigate whether severity ratings decreased with time for participants or for raters. The correlations of event age with the PCL sum and status measures and with the self-rated severity measures were not significant. The correlations of event age with the other ratings of the Four Kinds of Damage, Versus Other Events, and Holmes-Rahe severity were $.18(p<.001), .21$ $(p<.001)$, and $.16(p<.01)$, respectively, indicating that our raters found older events to be somewhat more 
severe, even though the participants did not. As an added check that the age of the event was not having a substantial effect on our results, the PCL sum and status measures were predicted in multiple regressions simultaneously by event age and the three other-rated severity measures. None of predictors were significant at the $.05 p$ level. In stepwise regressions at the $.05 p$ level, the Holmes-Rahe entered for the PCL status measure, as expected from its simple correlation, and no other measures did. Thus, the age of the event does not seem to be influencing our conclusions.

\section{Discussion}

Study 2 replicated the findings of Study 1 and demonstrated substantial overlap among self- and other-rated measures of severity. The only difference was that the PCL status measure correlated with the Holmes-Rahe, Four Kinds of Damage, and Other-Rated A1 severity. Given repeatable but somewhat puzzling findings in an unscreened population, we investigated only participants who had an A-criterion trauma as their negative event in Studies 3 and 4 .

\section{Study 3: Longer A-Criterion Event Narratives in a Clinical Sample}

\section{Metbod}

Participants. Undergraduates were recruited either from signs on campus or from their responses on a webbased prescreen. To ensure that our comparisons would be among trauma memories that met the current diagnostic criterion, we included participants only if an experienced, master's-level clinician judged that their trauma met the A trauma criterion of the DSM-IV-TR. The PCL was always rated on the trauma that was described. All participants in the PTSD group also met the remaining DSM-IV-TR criterion for current PTSD, which was assessed by a clinician using an individually administered CAPS. Participants were 30 Duke University undergraduates between the ages of 18 and 22, 15 with PTSD and 15 without. Although there was no overlap in PTSD symptom severity in the two groups, there was a continuous distribution of PCL scores to use in analyses. The traumas described in the participants' narratives, which were the traumas on which the diagnoses were made, could be grouped into the following categories, with the number of PTSD and non-PTSD participants in each category listed after each category name: sexual assault, rape, or childhood sexual assault, 6, 1; other serious crime, 3, 0; suicide or attempted suicide of a parent, other family member, or friend, 2, 3; other death of a parent, sibling, other family member, or friend; 1, 6; sudden serious or life-threatening accident or illness to participant, sibling, or parent, 3, 5. The trauma categories were fairly well matched across the two groups. Any differences were in the direction of somewhat more serious traumas in the PTSD group, which would tend to produce correlations between event severity and symptom severity.

Procedure. The materials, rating procedures, and raters were identical to those used in Study 1. The experimental procedure was conducted within a few days of the clinical diagnosis for the participants with PTSD. Participants were tested individually. They began by nominating and providing a one-line description of three memories, including "the most stressful or traumatic event in your life that now bothers you the most, with the restriction that it occurred one month or longer ago" and two comparison memories, which were not used here. The participants were then given a separate page to describe each event. In contrast to the request in Studies 1 and 2 to provide a short description, here participants recorded a one-page description of their stressful event. Thus, the descriptions were much more detailed than those in the other experiments. The order of events was randomized with the restriction that the traumatic event could not be last. This was done to minimize any lingering negative thoughts beyond the experimental session. The participants rated memories on several scales including the A1 and A2 criteria as dichotomous scales and then completed the CES, BDI-II, and PCL. For a full report, see Rubin (2011).

\section{Ratings}

Study 3 had the same rating procedures and raters as Studies 1.

\section{Results}

There was excellent agreement among the three otherrated severity measures given the restricted range of severity caused by all 30 negative events being A traumas. The Six Kinds of Damage severity correlated with the Versus Other Events and Holmes-Rahe severity at .69 and .60, respectively. The Versus Other Events and Holmes-Rahe severity correlated at .74 (all ps < .001). Thus, we have converging measures of severity. The PCL correlated in reasonable ways with the individual differences measures. As shown in Table 3, the other-rated severity measures correlated neither with the PCL nor with the clinical diagnosis. Here the Self-Rated A2 did not show the relation to the PCL it did in Studies 1 and 2. A 
possible reason is that all participants had to have evidence of an A-criterion trauma to be included and so had experienced an event they could rate that had strong A2 emotions.

\section{Discussion}

In three studies with undergraduates, we consistently found internally consistent measures of objective event severity that did not correlate with PTSD symptom severity. To ensure these results would generalize to another population, we included clinically diagnosed, community-dwelling adults, with a larger range of traumas.

\section{Study 4: A Community-Dwelling Clinical Sample}

\section{Metbod}

Materials. The NEO (Costa \& McCrae, 1992) provides a comprehensive assessment of adult personality with five domains. Individuals make judgments about typical past actions or thoughts in order to agree or disagree with a series of 240 statements. The personality description provided is based on personal semantic memory. The scales for the domains are all $t$ scores based on standardized norms; thus, 50 is the mean and 10 is the standard deviation of the standard comparison population.

The TLEQ (Kubany et al., 2000) was developed as a way of reminding people of possible traumas to get a more complete reporting by giving a series of 23 classes of possible traumas. Participants indicated whether the
A1 and A2 PTSD criteria were met for traumas they reported.

Participants and methods. Adults from the community were screened by a master's-level clinician who was trained and worked regularly in a research setting. Participants were recruited via advertising for a study on memory for stressful or traumatic events and how they differ from more everyday memories. The CAPS was used to determine PTSD diagnostic status.

Current diagnoses were determined by a 1-month time frame for PTSD. Any potential participants meeting criteria for current alcohol or other substance dependence/ abuse or psychotic disorders based on clinical interviews were excluded. Participants were also excluded if they were medically unstable or if they could not complete the study procedures. This resulted in 75 participants ( 44 women, 31 men, mean age of 47). Their index traumas, followed by the number of participants in each category, were childhood physical or sexual abuse, 9; other childhood violence, 5; adult physical or sexual assault, 11; adult domestic violence, 2; combat, 8; other adult violence, 9; accident, 2; death of a family member or friend, 12; and other, 15. There were three sessions in the entire procedure. The BDI-II, CAPS, CES, PCL, and TLEQ were given in the initial session; the NEO was given in a later session.

\section{Ratings}

Study 4 had the same rating procedures and raters as Studies 1 and 3 . We used both the participant's brief

Table 3. Study 3 Means and Correlations With PCL and PTSD Diagnosis

\begin{tabular}{|c|c|c|c|c|c|}
\hline \multirow[b]{2}{*}{ Variable } & \multirow[b]{2}{*}{$M$} & \multirow[b]{2}{*}{$S D$} & \multirow[b]{2}{*}{$\alpha$} & \multicolumn{2}{|c|}{$r(28)$} \\
\hline & & & & PCL & Diagnosis \\
\hline \multicolumn{6}{|c|}{ Other-rated general severity } \\
\hline Versus Other Events & 2.98 & 1.01 & .74 & .02 & .10 \\
\hline Holmes-Rahe Scale & 47.61 & 10.33 & .91 & -.05 & -.02 \\
\hline Six Kinds of Damage & 2.58 & 1.02 & .94 & -.29 & -.21 \\
\hline \multicolumn{6}{|c|}{ Self-rated $D S M-I V$ severity } \\
\hline A1 & 0.87 & 0.35 & - & -.05 & .00 \\
\hline A2 & 0.93 & 0.25 & - & .01 & .00 \\
\hline \multicolumn{6}{|c|}{ Individual differences } \\
\hline CES & 4.10 & 0.83 & .90 & .19 & .22 \\
\hline BDI-II & 11.50 & 9.72 & .93 & $.78^{* * * * * *}$ & $.77^{* * * * * * * * 6}$ \\
\hline PCL sum & 40.03 & 16.18 & .94 & - & $.95^{\text {******** }}$ \\
\hline PCL diagnosis (\%) & 50.00 & 50.86 & - & $.95^{* * * * *}$ & - \\
\hline
\end{tabular}

Note: $n=30$. PCL $=$ PTSD Checklist CES $=$ Centrality of Event Scale; BDI-II = Beck Depression Inventory-II.

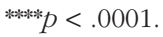


description and the clinician's CAPS notes description for the other-rated severity measures.

\section{Results}

There was excellent agreement among the three otherrated severity measures, especially given that all traumas met the A criterion. The Six Kinds of Damage severity correlated with the Versus Other Events and HolmesRahe severity at .67 and .65 , respectively. The Versus Other Events and Holmes-Rahe severity correlated at .73 (all $p s<.0001$ ). Thus, we have converging measures of severity from three different perspectives, and the PCL correlated with individual differences measures as expected. However, as shown in Table 4, the other-rated severity measures did not predict the PCL.

\section{Discussion}

Study 4 replicated the basic findings of the earlier three studies in a community-dwelling population, excluding the possibility that our findings were restricted to undergraduates or those with milder negative events or traumas. In a sample of individuals all of whom were diagnosed with PTSD, there was still enough variation in the severity of traumas and in the severity of PTSD symptoms to produce correlations among our three severity measures and between the PCL and the CES, BDI-II, and neuroticism similar to those in the earlier three studies.

Table 4. Study 4 Means and Correlations With PCL

\begin{tabular}{|c|c|c|c|c|}
\hline Variable & $M$ & $S D$ & $\alpha$ & $\begin{array}{c}r \text { with } \\
\text { PCL }\end{array}$ \\
\hline \multicolumn{5}{|c|}{ Other-rated general severity } \\
\hline Versus Other Events & 3.50 & 1.00 & .67 & .05 \\
\hline Holmes-Rahe Scale & 54.20 & 10.16 & .89 & -.04 \\
\hline$\underline{\text { Six Kinds of Damage }}$ & 2.70 & 1.07 & .92 & .04 \\
\hline \multicolumn{5}{|c|}{ Individual differences } \\
\hline CES & 4.00 & 0.78 & .89 & .20 \\
\hline BDI-II & 21.25 & 12.21 & .93 & $.58^{* * * * * * *}$ \\
\hline NEO N & 54.57 & 10.23 & - & $.35^{* *}$ \\
\hline NEO E & 40.73 & 10.68 & - & $-.28^{*}$ \\
\hline NEO O & 46.94 & 5.93 & - & .02 \\
\hline NEO A & 52.29 & 10.94 & - & $-.24^{*}$ \\
\hline NEO C & 48.54 & 9.10 & - & $-.23^{*}$ \\
\hline TLEQ A only & 10.09 & 4.04 & - & $.24^{*}$ \\
\hline TLEQ all & 11.59 & 3.90 & - & .17 \\
\hline PCL sum & 49.10 & 15.69 & .94 & - \\
\hline
\end{tabular}

Note: $n=75$, except for the NEO scales, for which $n=73$. $\mathrm{PCL}=$ PTSD Checklist; CES = Centrality of Event Scale; BDI-II = Beck Depression Inventory-II; NEO = NEO Personality Inventory; N, E, O, $\mathrm{A}$, and $\mathrm{C}=$ neuroticism, extroversion, openness, agreeableness, and conscientiousness; TLEQ $=$ Traumatic Life Events Questionnaire. ${ }^{*} p<.05 .{ }^{* *} p<.01 . * * * * * 0001$.
In the original study from which these data were drawn (Rubin, Dennis, \& Beckham, 2011), there was a control group without PTSD in which 37 participants had detailed enough descriptions to support severity ratings. For the purposes of the original study in which clearly distinct and nonoverlapping samples were desired, the control participants were not screened to have an A-criterion trauma, could not have symptoms that were close to meeting a PTSD diagnosis (a subthreshold PTSD exclusion), and could not have a trauma that was reported to have once produced PTSD symptoms (a lifetime PTSD exclusion). Combining these two groups into a single analysis would have therefore favored a relationship between symptom severity and trauma severity, even if it was not present in the general population from which the samples were drawn because we would be mixing participants with both symptom severity and event severity high enough to meet the PTSD criteria with participants screened for lower symptom severity who were not required to have traumas severe enough to meet the $\mathrm{A}$ criterion. In more quantitative terms, in Studies 1 and 2 our other-rated severity measures correlated at about .5 with our other-rated A1 measure done by clinically trained raters. Thus, comparing groups with and without an A1 trauma should result in differences in general event severity. Thus, when the A1 criterion is confounded with PTSD severity, even without the enhanced differences in PTSD symptom severity in the original study, it could produce spurious correlations between event severity and symptom severity.

Nonetheless, as a check to see whether we could see effects of trauma severity on symptom severity when the control group was included, we did an exploratory analysis including the control participants. There were still no significant correlations between the PCL sum and the three measures of trauma severity. There were, however, significant correlations between the clinical diagnosis on which participants were screened and two of our three measures of severity. The Versus Other Events and Holmes-Rahe severity both had $r(110)$ values of .24 ( $p$ s < $.05)$; the Six Kinds of Damage had an $r(110)$ of .08 ( $p=$ .42 ). Thus, even with groups biased to enhance a correlation of trauma severity and PTSD and PTSD symptom severity, the relationship was not strong. In contrast, Study 3 participants all had A-criterion traumas, and controls were not excluded for subthreshold or lifetime PTSD. Their data resulted in no relationship between trauma severity and PTSD or PTSD symptom severity.

\section{General Discussion}

We formulated three neutral observer measures of the general severity of events based on different theoretical frameworks and different rating methods, and compared 
them to the A1 criterion measure and to similar measures rated by the participants as well as to PTSD symptom severity. Because we knew of no other studies of PTSD symptom severity that had raters measuring general severity and because the results of our first study were not expected, we replicated our findings in a total of four samples. Two samples were undergraduates unselected for symptom severity or event severity, and two were clinically diagnosed participants with A-criterion traumas. We repeatedly obtained the same basic findings: Both other-rated severity and PTSD symptom severity correlated in expected and reasonable ways with a host of measures including ratings of event severity made by the participants themselves. However, symptom severity and other-rated event severity did not reliably correlate with each other.

The results in terms of our three hypotheses were as follows. First, we expected and found that our three other-rated measures of general severity correlated substantially, and so we were able to measure event severity as a unitary concept. Second, we expected and found that our measures of general event severity correlated with similar measures of self-rated event severity, which further supported the validity of these measures. Third, and counter to our expectations, our measures of general event severity did not correlate with PTSD symptom severity.

Consider three nonexclusive explanations of our results. The first alternative, which is consistent with our data and the general literature, is the most radical. It is that the severity of an event as judged as objectively as possible by outside observers does not affect PTSD symptom severity, though it does correlate with the person's own judgment of the severity of the event. A weaker version of this alternative, in which a person's perception of his or her trauma is an important contributor to PTSD symptoms beyond any effect of the objective trauma, is widely held by cognitive theories of PTSD (e.g., Bryant, 2011; Hembree \& Foa, 2010). Moreover, this weaker version is assumed by all therapies that have patients reinterpret the severity, impact, coherence, integration into the life story, or meaning of traumas (e.g., Brewin \& Holmes, 2003; Dalgleish, 2004; Ehlers \& Clark, 2000).

The second alternative is that it is not the overall measure of general severity that matters. Rather, different categories of traumas produce differing degrees of PTSD symptom severity. Thus, as noted in the DSM-III quote given earlier, torture and a motor vehicle accident that are judged by the person to whom they occurred, or by an outside observer, or by any other means to have equal severity may cause different levels of PTSD symptom severity. Within each category severity may play a role, but across categories the effects of a trauma must be determined by measures other than general severity. Under this alternative, the challenge is to define the categories and their relative effects on PTSD symptom severity. Evidence exists for both differences in categories and severity within categories, as reviewed in the introduction. Obtaining empirical support for choosing particular categories and measuring severity within each one will not be an easy task. Another approach in the same spirit would be to develop a typology of traumatic events (Dohrenwend, 2010). Instead of categories of events, there would be dimensional characteristics of events including the degree to which the source of the trauma was external to behavior of the individual or caused by it, valence, unpredictability, magnitude, centrality to goals, plans, and concerns, and tendency to exhaust the individual physically. Again, obtaining empirical support for the weighting of dimensions will not be an easy task. For both the category and typology approach, the combination of components will have to yield information not obvious to the implicit mental calculations of our raters.

The third alternative is that the focus on a single trauma ignores the cumulative effect of multiple traumatic or near traumatic events, effects that could exceed and mask those of the severity of the index trauma. The standard developmental approach in psychology is to assume that people's current state is affected by their genetic disposition and their history of environmental influences, including traumatic events, along their developmental trajectory. The focus on a single trauma in PTSD is a serious challenge to this productive and empirically well-supported approach. Thus, under the standard developmental approach, a veteran returning from war might be more affected by the cumulative and sequential effects of multiple trauma-like events occurring over his or her entire life than by the severity of any single event (e.g., Berntsen et al., 2012). Under this alternative, objective trauma severity could be crucial, but the entire history of such events rather than one trauma would need to be considered. It is also likely that particular developmental stages, such as childhood or adolescence, may be of special importance (Ogle, Rubin, \& Siegler, in press). A rough, retrospective index of this history may be seen in individual difference measures such as personality or coping styles or in counts of past traumas or types of traumas as noted here by the TLEQ correlations in Table 4 as well as in the literature in general (e.g., Neuner et al., 2004).

Converging evidence that other-rated severity does not account for psychological differences in situations where it might be expected to comes from studies outside of PTSD, studies that use as detailed a procedure to measure the general severity of negative events as seems 
practically possible. In their studies, Hammen and colleagues (Espejo et al., 2011; Hammen, 1991) had interviewers elicit descriptions of events from participants' lives with the goal of obtaining enough information about each event to characterize its impact, including what occurred and for how long, what resources and preparation the participants had to handle the event, consequences of the event, and other categories of information. Evaluative comments were removed from the descriptions before independent raters indicated how much impact the events would have on a typical person on a scale similar to our Versus Other Events severity rating. Thus, a much more comprehensive, a more laborintensive, and, for the extremely negative and traumatic memories used here, a potentially more intrusive procedure was used for the elicitation, development, and rating of the narratives.

Using this procedure, Espejo et al. (2011) had participants report on stressful events from the past 6 months. On two occasions separated by 2 weeks, participants rated the severity of their own events on the same scale raters used, but with reference to themselves instead of a typical person. This allowed the comparison of self-rated and other-rated severity. In both Espejo et al. and our Study 2, self- and other-rated severity correlated, but only self-rated severity was related to the measures of interest. In particular, in Espejo et al., the other ratings from the two occasions correlated with the self-ratings at .28 and .28 , and these were the only significant correlations otherrated severity had. In contrast, the self-rated severity taken at two occasions also correlated with neuroticism at .31 and .26 and with negative affect at .47 and .28. In an earlier longitudinal study using the same careful other-rated severity method, Hammen (1991) demonstrated that the total severity of negative events occurring over a 1-year period was different in unipolar, bipolar, chronic medical, and control groups for both all kinds of negative events and theoretically relevant categories of negative events. However, similar results were obtained when a simple frequency count of such events was used rather than summing the severity of the events. Thus, the findings from extremely thorough productions of narratives do not appear to fare better than the results obtained here with simpler narratives and do not point to obtaining results that would lead to different theoretical conclusions.

\section{Limitations}

One limitation is that we did not measure in a systematic way risk factors that could affect PTSD symptom severity (e.g., Brewin et al., 2000). Those that we did measure, such as neuroticism, did have the expected correlations. If our other-rated severity measures had fared better, such risk factors could have added predictive value to them. Even with the current findings, it is possible that otherrated severity measures could have modulated the correlations between risk factors and symptom severity such that risk factors could have played more of a role when the traumatic event was less severe (McNally \& Robinaugh, 2011; but see Breslau, Troost, Bohnert, \& Luo, 2012).

Another limitation is the absence of any consideration of allostatic load, which results from chronic affects at regulation in the face of stressful events (McEwen \& Gianaros, 2011). We considered as one possible reason for our failure to find a relationship between other-rated severity and symptom severity the possibility that cumulative effects of many traumatic events rather than just the single trauma on which severity was measured was what mattered. However, there are stressors not based on specific events that add to allostatic load.

In addition, after failing to find instruments to measure general severity, we devised our own based on methods that would use different approaches and would work empirically. We did not try to adapt existing measures from the literature on stress. For example, Hobfoll (2001) proposed a measure of stress based on the loss or threat of loss of resources, much of which would correspond to our Six Kinds of Damage, but was based on a more coherent theoretical analysis.

\section{Conclusions}

Our first hypothesis was that our three other-rated measures of general severity, each taken from a different approach and using a different method to define severity, would correlate substantially. It was confirmed, allowing us to consider our three measures of event severity to be measuring a single concept. This has important methodological and theoretical implications. Methodologically, we have introduced into the literature a novel set of three measures of other-rated general event severity that have been shown to be correlated highly enough that they can be considered to be measuring the same concept. Theoretically, given the face validity of the measures, it is difficult to imagine other objective measures of general event severity that would behave in a dramatically different manner. Our second hypothesis that our measures of general event severity would correlate with similar measures of self-rated event severity was also confirmed and further supports the value of our other-rated measures.

Our third hypothesis was that we expected our measures of general event severity to predict PTSD symptom severity, at least to a modest degree. The failure to find support for this hypothesis has serious practical and theoretical implications. At a practical level, the current diagnosis relies on there being an objective traumatic event 
that meets defined severity criteria as determined by a trained, licensed clinician who is an outside observer and not by the person being diagnosed or by that person's reaction to an unspecified negative situation. Data arguing that outside observer ratings of the event are not useful predictors of clinically relevant outcomes should add to questions about this aspect of the diagnosis. Given the lack of substantial correlation with the DSM-IV-TR A1 severity measure used in Studies 1 and 2, it is not clear that the $D S M-5$ definition of severity will do better. Theoretically, if the other-rated severity of a single event does not correlate at least modestly and reliably with symptom severity, then we need either to find a more predictive measure of event severity or to develop other theoretical explanations for the symptoms that arise in PTSD. We do not and cannot claim that there was no relation between the other-rated event severity of a single trauma and PTSD symptom severity, but it is at best small and not reliable even with fairly large samples, which makes it unlikely that the relation plays a major causal role in PTSD.

It would have been simpler practically and theoretically if we had found that a general measure of objective severity was a substantial determiner of PTSD symptom severity. However, the agreement among our three measures of general severity and their lack of correlation with PTSD symptom severity make this solution less likely. In contrast, the cases for differences between categories of traumas, for differential effects occurring as a function of the developmental stage at the trauma, and for the cumulative effects of traumas are substantial. Our data combined with this existing literature suggest that it may not be optimal to measure the severity of only one trauma, even the index trauma, rather than beginning the detailed work needed to develop models of how specific kinds of traumas experienced at specific times in the developmental trajectory contribute to cumulative PTSD severity.

This is not a simple request for more research. If our results hold up to scrutiny and replication, that is, if objective measures of the severity of the index trauma on which the diagnosis is made have little or no effect on symptom severity, the basic theoretical assumptions of the diagnosis will be challenged. The current emphasis on the A-criterion trauma might still serve a useful purpose in system of differential diagnosis, but it would not lead to an understanding of the disorder itself. Instead of the emphasis on a relatively easy-to-conceptualize single external index trauma, the entire developmental history including traumatic events and their types would be needed. If the results here hold, the path will be more difficult but perhaps more fruitful.

\section{Acknowledgments}

We wish to thank Dorthe Berntsen for comments and Katie Barzee, Natalie Buse, and Ivy Paw for their work on developing the ratings.

\section{Declaration of Conflicting Interests}

The authors declared that they had no conflicts of interest with respect to their authorship or the publication of this article.

\section{Funding}

This work was supported by the National Institute of Mental Health (Grant R01 MH066079).

\section{References}

American Psychiatric Association. (1980). Diagnostic and statistical manual of mental disorders (3rd ed.). Washington, DC: Author.

American Psychiatric Association. (2000). Diagnostic and statistical manual of mental disorders (4th ed., text revision). Washington, DC: Author.

Beck, A. T., Steer, R. A., \& Brown, G. K. (1996). Manual for the Beck Depression Inventory-II. San Antonio, TX: Psychological Corporation.

Berntsen, D., Johannessen, K. B., Thomsen, Y. D., Bertelsen, M., Hoyle, R. H., \& Rubin, D. C. (2012). Peace and war: Trajectories of posttraumatic stress disorder symptoms before, during and after military deployment in Afghanistan. Psychological Science. Advance online publication. doi:10.1177/0956797612457389

Berntsen, D., \& Rubin, D. C. (2006). The Centrality of Event Scale: A measure of integrating a trauma into one's identity and its relation to post-traumatic stress disorder symptoms. Behaviour Research and Therapy, 44, 219-231.

Berntsen, D., \& Rubin, D. C. (2007). When a trauma becomes a key to identity: Enhanced integration of trauma memories predicts posttraumatic stress disorder symptoms. Applied Cognitive Psychology, 21, 417-431.

Berntsen, D., \& Rubin, D. C. (2008). The reappearance hypothesis revisited: Recurrent involuntary memories after traumatic events and in everyday life. Memory \& Cognition, 36, 449-460.

Blake, D. D., Weathers, F. W., Nagy, L. M., Kaloupek, D. G., Gusman, F. D., Charney, D. S., \& Keane, T. M. (1995). The development of a clinician-administered posttraumatic stress disorder scale. Journal of Traumatic Stress, 8, 75-80.

Blanchard, E. B., Jones-Alexander, J., Buckley, T. C., \& Foneris, C. A. (1996). Psychometric properties of the PTSD Checklist (PCL). Behaviour, Research and Therapy, 34, 669-673.

Breslau, N. (2012). Posttraumatic syndromes and the problem of heterogeneity. In C. S. Widom (Ed.), Trauma, psychopathology and violence: Causes, correlates, or consequences (pp. 3-20). New York, NY: Oxford University Press.

Breslau, N., Davis, G. C., Andreski, P., \& Peterson, E. (1991). Traumatic events and posttraumatic stress disorder in an urban population of young adults. Archives of General Psychiatry, 48, 216-222.

Breslau, N., Troost, J. P., Bohnert, K., \& Luo, Z. (2012). Influence of predispositions on post-traumatic stress disorder: Does it vary by trauma severity? Psychological Medicine, 43, 381390. doi:10.1017/S0033291712001195

Brewin, C. R., Andrews, B., \& Valentine, J. D. (2000). Metaanalysis of risk factors for posttraumatic stress disorder in trauma-exposed adults. Journal of Counseling and Clinical Psychology, 68, 748-766. 
Brewin, C. R., \& Holmes, E. A. (2003). Psychological theories of posttraumatic stress disorder. Clinical Psychology Review, 23, 339-376.

Bryant, R. (2011). Psychological interventions for trauma exposure and PTSD. In D. J. Stein, M. J. Friedman, \& C. Blanco (Eds.), Post-traumatic stress disorder (pp. 171-202). Hoboken, NJ: Wiley-Blackwell.

Costa, P. T., Jr., \& McCrae, R. R. (1992). Revised NEO Personality Inventory (NEO-PIR) and the NEO Five-Factor Inventory (NEO-FFI) professional manual. Odessa, FL: Psychological Assessment Resources.

Dalgleish, T. (2004). Cognitive approaches to posttraumatic stress disorder: The evolution of multirepresentational theorizing. Psychological Bulletin, 130, 228-260.

Dedert, E. A., Green, K. T., Calhoun, P. S., Yoash-Gantz, R., Taber, K. H., Miller, M. M., . . . Beckham, J. C. (2009). Association of trauma exposure with psychiatric morbidity in military veterans who have served since September 11, 2001. Journal of Psychiatric Research , 43, 830-836.

Dohrenwend, B. P. (2010). Toward a typology of high-risk major stressful events and situations in posttraumatic stress disorder and related psychopathology. Psychological Injury and Law, 3, 89-99.

Ehlers, A., \& Clark, D. M. (2000). A cognitive model of posttraumatic stress disorder. Behaviour Research and Therapy, 38, 319-345.

Espejo, E. P., Ferriter, C. T., Hazel, N. A., Keenan-Miller, D., Hoffman, L. R., \& Hammen, C. (2011). Predictors of subjective ratings of stressor severity: The effects of current mood and neuroticism. Stress and Health, 27, 23-33.

Hammen, C. (1991). Generation of stress in the course of unipolar depression. Journal of Abnormal Psychology, 100, $555-561$.

Hembree, E. A., \& Foa, E. B. (2010). Cognitive behavioral therapies for PTSD. In G. M. Rosen \& C. B. Frueh (Eds.), Clinician's guide to posttraumatic stress disorder (pp. 177203). Hoboken, NJ: John Wiley.

Hobfoll, S. E. (2001). The influence of culture, community, and the nested-self in the stress process: Advancing conservation of resources theory. Applied Psychology, 50, 337-370.

Holmes, T. H., \& Rahe, R. H. (1967). The social readjustment rating scale. Journal of Psychosomatic Research, 11, 213218.

John, O., Donahue, E., \& Kentle, R. (1991). The "Big Five" Inventory-Versions $4 a$ and 54 (Technical report). Berkeley: University of California, Berkeley, Institute of Personality Assessment and Research.

John, O. P., \& Srivastava, S. (1999). The Big-Five trait taxonomy: History, measurement, and theoretical perspectives. In L. A. Pervin \& O. P. John (Eds.), Handbook of personality: Theory and research (Vol. 2, pp. 102-138). New York, NY: Guilford.

Kessler, R. C., Sonnega, A., Bromet, E., Hughes, M., \& Nelson, C. B. (1995). Posttraumatic stress disorder in the National Comorbidity Survey. Archives of General Psychiatry, 52, 1048-1060.
Kubany, E. S., Haynes, S. N., Leisen, M. B., Owens, J. A., Kaplan, A. S., Watson, S. B., \& Burns, K. (2000). Development and preliminary validation of a brief broad spectrum measure of trauma exposure: The Traumatic Life Events Questionnaire. Psychological Assessment, 12, 210-224.

Lancaster, S. L., Melka, S. E., \& Rodriguez, B. F. (2009). An examination of the differential effects of the experience of DSM-IV defined traumatic events and life stressors. Journal of Anxiety Disorders, 23, 711-717.

McEwen, B. S., \& Gianaros, P. J. (2011). Stress- and allostasisinduced brain plasticity. Annual Review of Medicine, 62, 431-435.

McNally, R. J., \& Robinaugh, D. J. (2011). Risk factors and posttraumatic stress disorder: Are they especially predictive following exposure to less sever stressors? Depression and Anxiety, 28, 1091-1096.

Neuner, F., Schauer, M., Karunakara, U., Klaschik, C., Robert, C., \& Elbert, T. (2004). Psychological trauma and evidence for enhanced vulnerability for posttraumatic stress disorder through previous trauma among West Nile refugees. $B M C$ Psychiatry, 4, 34-40.

Ogle, C. M., Rubin, D. C., \& Siegler, I. C. (in press). The impact of the developmental timing of trauma exposure on PTSD symptoms and psychosocial functioning among older adults. Developmental Psychology.

Rubin, D. C. (2011). The coherence of memories for trauma: Evidence from posttraumatic stress disorder. Consciousness and Cognition, 20, 857-865.

Rubin, D. C., Berntsen, D., \& Bohni, M. K. (2008). A memorybased model of posttraumatic stress disorder: Evaluating basic assumptions underlying the PTSD diagnosis. Psychological Review, 115, 985-1011.

Rubin, D. C., Boals, A., \& Berntsen, D. (2008). Memory in posttraumatic stress disorder: Properties of voluntary and involuntary, traumatic and non-traumatic autobiographical memories in people with and without PTSD symptoms. Journal of Experimental Psychology: General, 137, 591-614.

Rubin, D. C., Dennis, M. F., \& Beckham, J. C. (2011). Autobiographical memory for stressful events: The role of autobiographical memory in posttraumatic stress disorder. Consciousness and Cognition, 20, 840-856.

Smith, D. M. (2011). Diagnosing liability: The legal history of posttraumatic stress disorder. Temple Law Review, 84, $1-69$.

Weathers, F. W., Keane, T. M., \& Davidson, J. R. (2001). Clinician-Administered PTSD Scale: A review of the first ten years of research. Depression and Anxiety, 13, 132-156.

Weathers, F. W., Litz, B. T., Huska, J. A., \& Keane, T. M. (1994). The PTSD Checklist (PCL). Unpublished scale, National Center for PTSD, Boston, MA.

Weiss, D. S., Brunet, A., Best, S. R., Metzler, T. J., Liberman, A., Pole, N., . . Marmar, C. R. (2010). Frequency and severity approaches to indexing exposure to trauma: The critical incident history questionnaire for police officers. Journal of Traumatic Stress, 23, 734-743. 